\title{
Expression of DcR3 and Its Effects in Kaposi's Sarcoma-Associated Herpesvirus-Infected Human Endothelial Cells
}

\author{
Seungmin Yoo Jaehyuk Jang Sil Kim Haewol Cho Myung-Shin Lee \\ Department of Microbiology and Immunology, Eulji University School of Medicine, Daejeon, South Korea
}

\section{Key Words}

Kaposi's sarcoma-associated herpesvirus $\cdot$ Kaposi's

sarcoma $\cdot$ DcR3 $\cdot$ TL1A

\begin{abstract}
Objective: Kaposi's sarcoma-associated herpesvirus (KSHV) is classified as a gamma-herpesvirus and it causes Kaposi's sarcoma in patients infected with the human immunodeficiency virus (HIV). Decoy receptor 3 (DcR3) is known as a decoy receptor for Fas ligand, LIGHT and TL1A and it can neutralize the biological effect of TL1A by inhibiting the TL1ADR3 interaction in human endothelial cells. The present study examined the expression of DcR3 in human endothelial cells and its effect during the early stages of KSHV infection. Methods: The expression of DcR3 was assessed using real-time RT-PCR and ELISA in human umbilical cord vein endothelial cells (HUVECs) infected with KSHV. Cell proliferation and apoptosis of KSHV-infected HUVECs were assessed after treatment of infected cells with an anti-DcR3 antibody or recombinant human TL1A. Results: DcR3 expression was induced during the early phase of KSHV infection. Inhibition of DcR3 with anti-DcR3 antibodies or recombinant human TL1A-induced apoptosis in KSHV-infected HUVECs. Conclusion: The expression of DcR3 plays an important role in the prevention of apoptosis in HUVECs during the early phases of KSHV infection, thus ensuring the successful establishment and maintenance of the viral infection.
\end{abstract}

Copyright $\odot 2011$ S. Karger AG, Basel
(C) 2011 S. Karger AG, Basel

0300-5526/12/0551-0045\$38.00/0

Fax +41613061234

E-Mail karger@karger.ch

www.karger.com
Accessible online at: www.karger.com/int

\section{Introduction}

Kaposi's sarcoma (KS) is a vascular tumor, primarily consisting of proliferating spindle-shaped endothelial cells and infiltrates of inflammatory cells [1]. The human gammaherpesvirus KS-associated herpesvirus (KSHV), which was initially identified in a KS lesion from an AIDS patient [2], has been convincingly linked to the development of all four clinical forms of KS, including classical KS, AIDS-related KS, African endemic KS, and immunosuppressed post-transplant KS [3]. The genome of KSHV and its encoded gene products are detected in the majority of spindle cells and sporadically in monocytes, macrophages, keratinocytes, and lymphocytes in KS tumors, but not in adjacent tissues or control tissues from patients without KS. KSHV has also been associated with other lymphoproliferative diseases, including primary effusion lymphoma and multicentric Castleman's disease.

Decoy receptor 3 (DcR3) is a lymphotoxin-like member of the tumor necrotic factor receptor superfamily. In addition to acting as the decoy receptor for Fas ligand, DcR3 competes with herpes simplex virus glycoprotein $\mathrm{D}$ for binding to herpesvirus entry mediator, a receptor expressed by T lymphocytes (LIGHT), and with TNFlike cytokine 1A (TL1A) [4-6]. The binding of DcR3 to Fas ligand, LIGHT, and TL1A neutralizes the proapoptotic actions induced by the Fas-FasL, LIGHT-lymphotoxin- $\beta$, and TL1A-Death receptor3 interactions, respectively. DcR3 is overexpressed in cells derived from malig- 
nant tumors, such as those of the lung, colon, stomach, pancreas, and from gliomas [6-9]. Moreover, high serum levels of DcR3 have been detected in many cancer patients [10]. The overexpression of DcR3 may, therefore, constitute a relative advantage for tumor growth and survival. Supporting this notion, tumor cells engineered to release high amounts of DcR3 are protected from apoptotic cell death and chemotaxis, resulting in decreased immune cell infiltration in glioma xenografts [11].

DcR3 is upregulated in EBV-infected cells or EBV-associated lymphomas $[12,13]$. However, information on DcR3 levels in other human gammaherpesvirus-infected cells is limited. The aim of the present study was to investigate the expression and function of DcR3 in the KSHV infection of susceptible target cells such as human umbilical cord vein endothelial cells (HUVECs). The induction of cell proliferation, migration, tube formation and angiogenesis in HUVECs by DcR3 through interference with TL1A function [4] suggests that DcR3 could have a similar effect in KSHV-infected HUVECs when it is upregulated after infection. TL1A or TNF superfamily member 15 (TNFSF15) is also expressed in endothelial cells and soluble TL1A fragments can induce apoptosis of endothelial cells in an autocrine manner [14, 15]. In the present study, DcR3 expression was shown to increase in the immediate early phase of viral infection and to play a role in the inhibition of apoptosis in KSHV-infected HUVECs.

\section{Materials and Methods}

\section{Cells and Reagents}

Primary cultures of human umbilical vein endothelial cells (HUVEC; Clonetics, San Diego, Calif., USA) were subcultured in endothelial cell growth medium (EGM-2 BulletKit; Clonetics) containing human endothelial growth factor, human fibroblast growth factor B, vascular endothelial growth factor, ascorbic acid, hydrocortisone, long R3-IGF-1, and heparin as described by the manufacturer. BCBL-1 and 293 cells harboring the recombinant KSHV, BAC36, have been described previously [16, 17]. Anti-human DcR3 antibody and recombinant human TL1A were purchased from R\&D systems (Minneapolis, Minn., USA).

\section{Preparation and Infection of KSHV BAC36}

The recombinant KSHV BAC36, containing a green fluorescent protein (GFP) cassette, was induced in BCBL-1 cells or 293T cells harboring BAC36 by treating the cells with $20 \mathrm{ng} \mathrm{ml}^{-1}$ of tetradecanoyl phorbol acetate (TPA, Sigma, St. Louis, Mo., USA) for 3 days. The isolated virus was used to infect cells as described previously [18]. Infection was conducted by centrifugation at $2,500 \mathrm{~g}$ for $60 \mathrm{~min}$ with $5 \mu \mathrm{g} \mathrm{ml}^{-1}$ of polybrene (Sigma Aldrich). Infectivity was estimated by examining GFP expression under an inverted fluorescence microscope.
Immunofluorescence Assay

Cells were fixed with $4 \%$ paraformaldehyde for $10 \mathrm{~min}$, permeabilized with phosphate-buffered saline containing $0.3 \%$ Triton X-100 for $5 \mathrm{~min}$, and blocked with $5 \%$ bovine serum albumin (BSA). Cells were incubated with anti-ORF73 (Advanced Biotechnologies Inc., Columbia, Md., USA, 1:250) or anti-ORF59 (Advanced Biotechnologies Inc., 1:250) overnight at $4^{\circ}$ followed by incubation with rhodamine-labeled anti-rat antibody (KPL, UK) or rhodamine-labeled anti-mouse antibody (KPL, UK) for $30 \mathrm{~min}$ at room temperature. After washing, the sample was mounted with Vectashield ${ }^{\circledR}$ (Vector laboratories, Inc., Burlingame, Calif., USA) and stained with 4,6-diamidino-2-phenylindole (DAPI). Analyses were performed using a Nikon ECLIPSE E400 fluorescence microscope.

\section{Flow Cytometry}

KSHV-infected or mock-infected HUVECs were detached from the plate with $0.25 \%$ trypsin-EDTA, and flow cytometry was performed with a FACSCalibur flow cytometer. The data were analyzed with CellQuest Pro software (Becton Dickinson, Bedford, Mass., USA). Prior to analysis, all samples were gated to eliminate dead cells.

\section{ELISA}

The DcR 3 concentration in cell-culture medium was analyzed with a human Decoy Receptor 3 ELISA kit (BioVendor Laboratory Medicine) according to the manufacturer's instructions. The culture supernatant from $5 \times 10^{5}$ culture $(2 \mathrm{ml})$ cells was collected and stored at $-70^{\circ}$ before use.

\section{Real-Time RT-PCR}

Total RNA from infected HUVECs was prepared with TRI reagent as recommended by the manufacturer (Sigma). Reverse transcription and quantitative PCR were conducted as described previously [18]. All samples, including a non-template control and internal glyceraldehyde 3-phosphate dehydrogenase (GAPDH) amplification controls, were examined in triplicate for each primer pair. Data analysis was carried out as described previously [15]. The primers used in this study were: DcR3_F650 5'-TCAATGTGCCAGGCTCTTC-3', DcR3_R793 5'-GCCTCTTGATGGAGATGTCC-3', GAPDH_F6 5'-GAAGGTGAAGGTCGGAGTC-3', GAPDH_R231 5'-GAAGATGGTGATGGGATTTC-3', TL1A_F1193 5'-GTTGTGAGACAAACTCCCACACAG-3', TL1A_ R1359 5'-CGGAATGTGACCTGGGAGTAAATG-3', LIGHT_ F630 5'-TGATACAAGAGCGAAGGT-3', LIGHT_R820 5'-TTGGAGTAGATGTAGTAGTAGC-3'. The primers were synthesized by GENOTECH (South Korea).

\section{Cell Proliferation Assay and Apoptosis Assay}

After viral infection, the viral supernatant was removed and culture media with anti-DcR3 antibody or recombinant human TL1A were added to each well. After incubation for $24 \mathrm{~h}$, the infections were monitored by detecting GFP expression under an inverted fluorescence microscope. Cell viability and terminal deoxynucleotidyl transferase dUTP nick end labeling (TUNEL) staining were evaluated with the cell proliferation reagent WST-1 (Roche Applied Sciences, Indianapolis, Ind., USA) and the in situ cell death detection kit, POD (Roche Applied Sciences), respectively, according to the manufacturer's instructions. Briefly, after fixing the HUVECs with $4 \%$ paraformaldehyde/PBS for $1 \mathrm{~h}$, the 
GFP

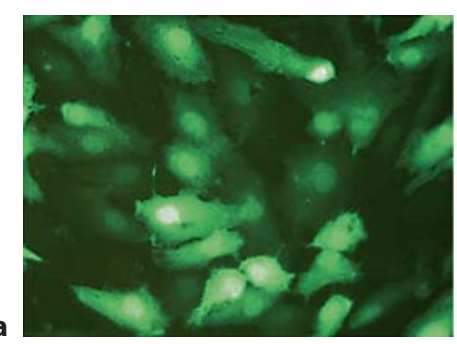

Fig. 1. KSHV infection of HUVECs. Subconfluent HUVECs were infected with KSHV BAC36 containing a GFP cassette. Microscopic observation of KSHV-infected HUVECs was performed $24 \mathrm{~h}$ after infection using a Nikon Eclipse E400 microscope equipped with a light source for fluorescence microscopy. a Expression of GFP and immunofluorescent detection of KSHV-infected HUVECs. KSHV-infected HUVECs were permeabilized, stained with anti-ORF73 antibody, and visualized with a rhodamine-labeled anti-rat antibody (original magnification $\times 400$ ). b Flow cytometric analysis of GFP expression in KSHV-infected HUVECs (gray). Mock-infected cells were overlaid as controls (white). c ORF73 and ORF59 expression of KSHV-infected HUVECs. HUVECs infected with KSHV at $24 \mathrm{~h}$ and $48 \mathrm{~h}$ postinfection were fixed onto glass slides, permeabilized, and incubated with primary rat $\mathrm{mAb}$ against KSHV ORF73, and primary mouse mAbs against KSHV ORF59. Cells were washed with PBS, followed by incubation with rhodamine-conjugated goat anti-rat immunoglobulin (IgG) or rhodimine-conjugated goat antimouse IgG. KSHV ORF73 and ORF59 staining is shown in red. Cells were visualized through a fluorescence microscope with $400 \times$ magnification.

cells were washed, incubated with $3 \% \mathrm{H}_{2} \mathrm{O}_{2}$ in methanol for 10 min, and then permeabilized with $0.1 \%$ Triton X-100/0.1\% sodium citrate for $2 \mathrm{~min}$ on ice. The samples were then rinsed three times with PBS and the TUNEL reaction was performed with TUNEL reaction mix according to the manufacturer's instruction at $37^{\circ}$ in a humidified chamber for $1 \mathrm{~h}$. After washing, converterPOD and DAB substrate were treated and samples were analyzed by Nikon ECLIPSE E400 light microscope. For DNA fragmentation analysis, the culture medium was removed and centrifuged at $2,500 \mathrm{~g}$ for $5 \mathrm{~min}$ to collect detached cells. The cell pellet was resuspended in $500 \mu \mathrm{l}$ of DNA extraction buffer $(50 \mathrm{mM}$ Tris, $\mathrm{pH}$ 8.0, $20 \mathrm{mM} \mathrm{Na}_{2}$ EDTA, $10 \mathrm{mM} \mathrm{NaCl}, 1 \%$ [w/v] SDS, and $20 \mu \mathrm{g}$ $\mathrm{ml}^{-1}$ RNase A [Sigma R-5503]) and incubated at $37^{\circ}$ for $1 \mathrm{~h}$, followed by the addition of proteinase K (Sigma P-2308) to a final concentration of $100 \mu \mathrm{g} \mathrm{ml}^{-1}$ and incubation at $65^{\circ}$ for $1 \mathrm{~h}$. The reaction containing DNA was purified once with chloroform/isoamyl alcohol (24:1) and precipitated with $5 \mathrm{M} \mathrm{NaCl}$. The precipitated DNA was electrophoretically separated on a $1 \%$ agarose gel containing $1 \mu \mathrm{g} \mathrm{ml}^{-1}$ ethidium bromide and visualized under ultraviolet transillumination.

DcR3 in KSHV-Infected Human Endothelial Cells

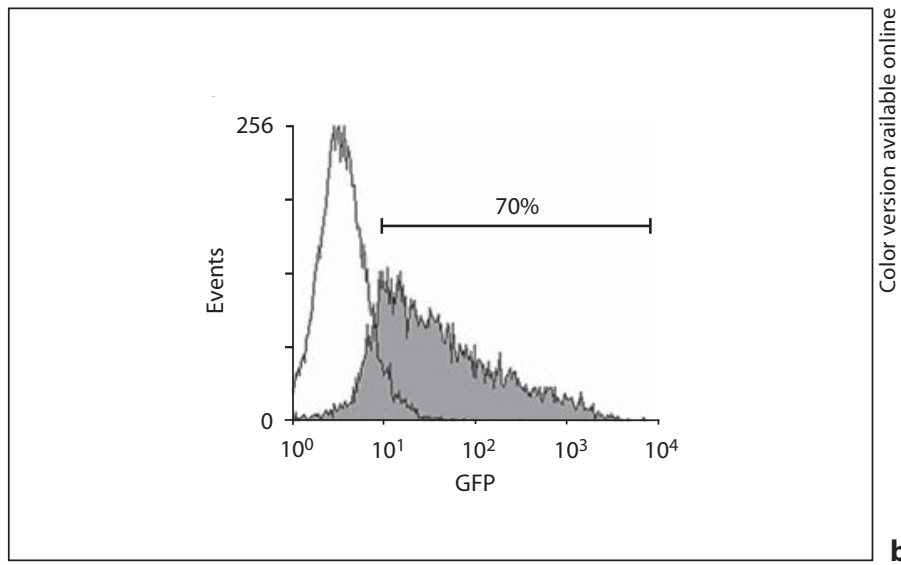

$24 \mathrm{~h}$
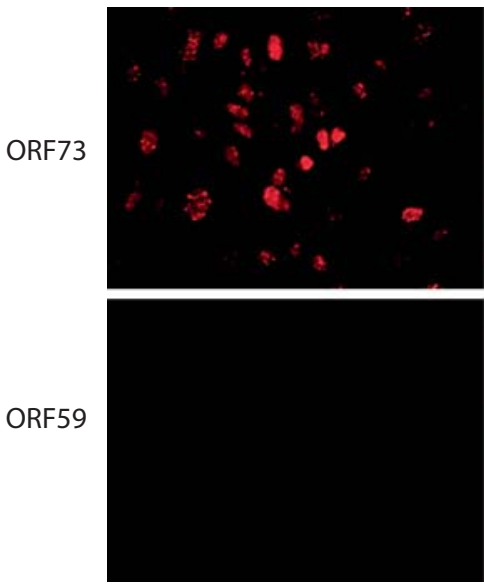

$48 \mathrm{~h}$
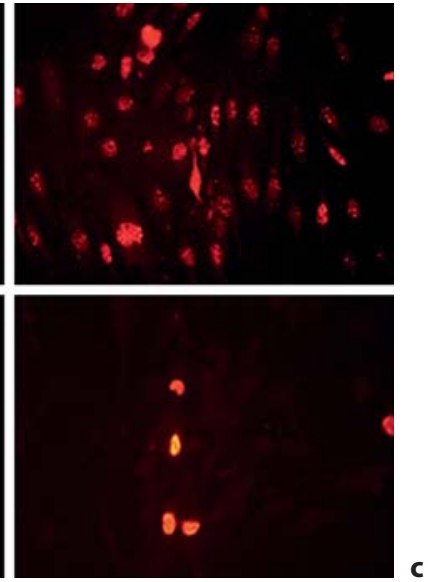

\section{Results}

\section{Infection of HUVECs with KSHV and Expression of DcR3}

After HUVECs were infected with the recombinant KSHV-BAC36 containing a GFP cassette by low-speed centrifugation [18], the infectivity was measured by monitoring GFP expression under a fluorescence microscope and the ratio of GFP-expressing cells was measured by flow cytometry (fig. 1). Fluorescence microscopy showed GFP expression in the infected cells, and most of the GFP-expressing cells also expressed ORF73 in the nucleus. At $24 \mathrm{~h}$ after infection, the overall expression rate was over 60\%; a representative figure is shown in figure $1 \mathrm{~b}$. To investigate lytic gene expression, immunofluorescence assay (IFA) was performed with antiORF73 and anti-ORF59 antibodies at 24 and $48 \mathrm{~h}$ after infection. The expression of ORF59, a KSHV lytic gene, 


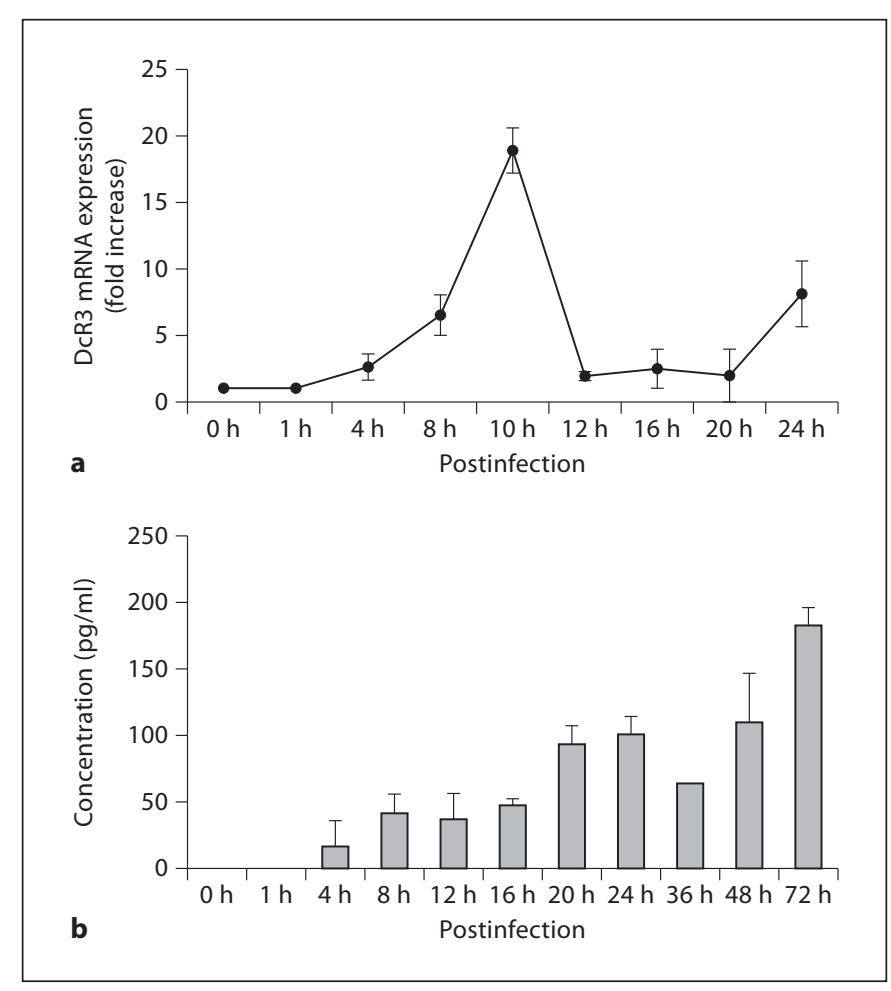

Fig. 2. Kinetics of DcR3 expression in the early phase of KSHV infection. Total RNA was isolated from KSHV-infected HUVECs and supernatants were collected at each time point. DcR3 mRNA and protein expression were assayed by quantitative real-time PCR and ELISA, respectively. a Relative expression of DcR3 mRNA in KSHV-infected HUVECs. The expression of DcR3 mRNA was calculated as described in Materials and Methods. The graph shows the relative mRNA expression at each time point based on the maximum expression measured. $\mathbf{b}$ Expression of soluble DcR3 protein in the supernatants of infected cells. The culture supernatant was collected at each time point and the concentration of DcR3 was measured by ELISA. All results represent the means of three independent experiments and standard deviations are indicated with error bars.

was not detected at $24 \mathrm{~h}$ but was detected at $48 \mathrm{~h}$ (fig. 1c). Our results are consistent with previous studies which showed that Orf59 mRNA is expressed $16 \mathrm{~h}$ after infection and that lytic protein is detected 2 days postinfection $[15,16]$. The DcR3 mRNA expression level in KSHVBAC36 infected HUVECs was tested at different time points by reverse transcription quantitative PCR methods (fig. 2a). DcR3 mRNA was detected $4 \mathrm{~h}$ after infection, and a significant increase in expression at 6 to $12 \mathrm{~h}$ postinfection and a decrease at $16 \mathrm{~h}$ were observed. After $24 \mathrm{~h}$, the expression level of DcR3 increased gradually again. The culture supernatant was also tested for DcR3 expression after KSHV infection (fig. 2b). At $4 \mathrm{~h}$ postin- fection, DcR3 began to be expressed and the concentration of DcR3 gradually increased until $72 \mathrm{~h}$ postinfection. These results indicate that KSHV is one of the factors causing the upregulation of DcR3 in HUVECs and that the expression of DcR3 is regulated differently depending on the length of time after KSHV infection.

\section{Effects of DcR3 in the Early Phase of KSHV Infection}

To investigate the effect of DcR3 in KSHV infection, an anti-DcR3 antibody was added to the KSHV-infected HUVECs. After $1 \mathrm{~h}$ of centrifugal infection, the virus was removed and EGM-2 containing anti-DcR3 or control IgG was added to the infected cells, followed by $24 \mathrm{~h}$ of culture. At $24 \mathrm{~h}$ postinfection, a cell proliferation assay showed significant differences in cell viability between the anti-DcR3 and the control IgG-treated groups, especially at antibody concentrations over $0.5 \mu \mathrm{g} \mathrm{ml}^{-1}$ (fig. 3a). As anti-DcR3 did not show any effects in mock-infected HUVECs, this inhibitory effect was not considered a result of the adverse effect of the anti-DcR3 antibody. Due to the potential presence of growth factors in the EGM-2 culture media, which could inhibit cell death even when DcR3 is blocked, the assay was repeated in serum and supplement-free endothelial basal medium-2 (fig. 3b). The cell viability pattern and the effect of anti-DcR3 were similar to those observed in EGM-2 culture medium, although overall cell viability was low in serum and supplement-free media. These results suggest that DcR3 produced in the early phase of KSHV infection of HUVECs inhibits cell death. Recombinant human TL1A, a ligand for DcR3, was serially diluted from a stock solution of 500 $\mathrm{ng} \mathrm{ml^{-1 }}$ and the dilutions were applied to KSHV-infected HUVECs in endothelial cell culture media with or without serum and supplements. TL1A showed a close association with cytotoxicity after KSHV infection regardless of the culture media used. Exposure to the recombinant human TL1A enhanced cytotoxic effects in the early phase of KSHV-infection of HUVECs but not in the early stages of mock infection of HUVECs, indicating that DcR3 protects against KSHV infection (fig. 4). As the recombinant TL1AL72-L251 used in these experiments has no effect on HUVEC proliferation or apoptosis [15], these results show that DcR3 protects against KSHV infection.

\section{DcR3 Inhibits Apoptosis during the Early Phase of KSHV Infection}

To understand the mechanisms underlying the effect of DcR3 on the inhibition of cell death in KSHV-infected HUVECs, two kinds of apoptosis assays were performed, namely the TUNEL staining assay and the DNA ladder 


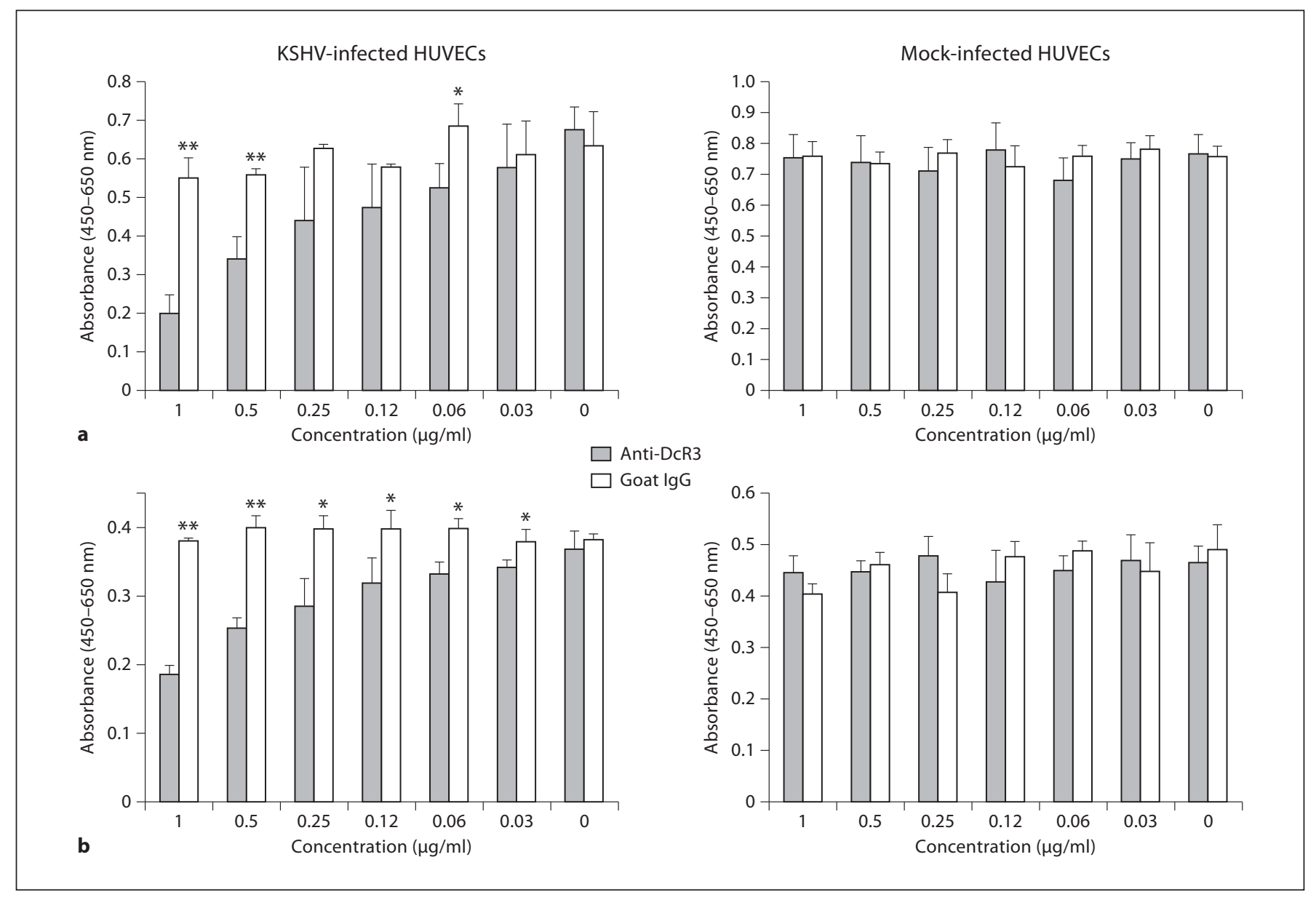

Fig. 3. Cytotoxic effect of anti-DcR3 antibody in KSHV-infected HUVECs. Anti-DcR3 antibody or control IgG antibody was applied to KSHV-infected HUVECs in 96-well culture plates immediately after infection of KSHV. Each antibody was added at various concentrations. After $24 \mathrm{~h}$ of incubation in endothelial growth medium with (a) or without serum (b), cell viability was tested with the cell proliferation reagent WST-1. Mock-infected HUVECs were used in control experiments. These results represent the means of triplicate experiments, and standard deviations are indicated with error bars. ${ }^{*} \mathrm{p}<0.05 ;{ }^{* *} \mathrm{p}<0.01$, anti-DcR3 antibody vs. control IgG. formation assay. As shown in figure $5 \mathrm{~A}$, control IgGtreated KSHV-infected HUVECs showed minimal TUNEL-positive staining, whereas anti-DcR3-treated cells displayed a significant proportion of TUNEL-positive apoptotic cells. An evaluation of the rate of apoptosis in anti-DcR3 treated cells was not possible due to the overall poor status of the cells and continuous cell death after infection. The results obtained with the apoptosis assays were consistent with the results of the cell proliferation assay described above. Cells from the same experiments were subjected to a DNA fragmentation assay (fig. 5B). Although DNA ladder formation was minimal in cells treated with control IgG, increased DNA laddering was observed in anti-DcR3-treated cells. Therefore, DcR3 produced by KSHV-infected HUVECs provides a survival advantage during the early phase of KSHV infection through an anti-apoptotic effect.

\section{Discussion}

Viruses have developed many mechanisms to hijack the metabolic pathways of their hosts for their own benefit and to evade attacks by the immune system. EBV and HTLV-1 use DcR3 to evade the defenses of the immune system during lymphomagenesis, and virus-infected DcR3-expressing lymphoma cells can also be selected during the multistep tumorigenesis process [15]. KSHV, 

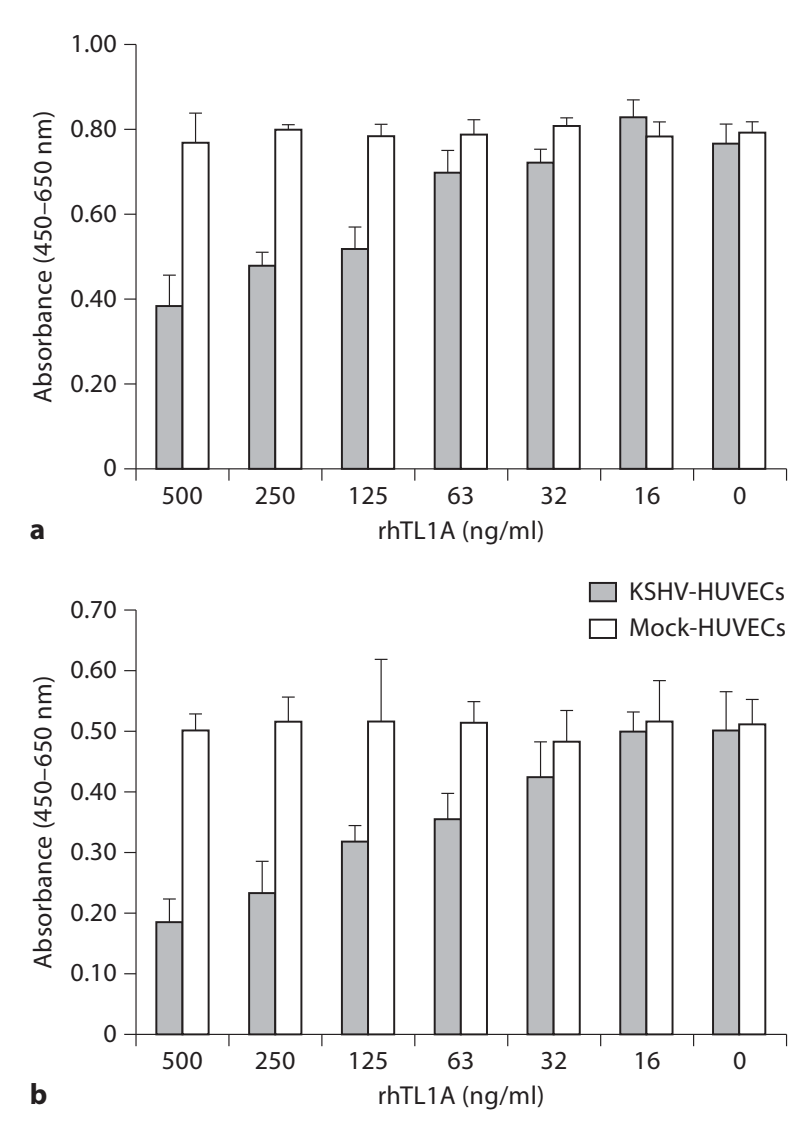

Fig. 4. Cytotoxic effect of exogenous TL1A in KSHV-infected HUVECs. HUVECs cultured in 96-well plates were treated with various concentration of TL1A immediately after KSHV infection. After $24 \mathrm{~h}$ of incubation in endothelial growth medium with (a) or without serum (b), cell viability was tested with the cell proliferation reagent WST-1. These assays were performed in triplicate and the mean values are shown.

also known as human herpesvirus 8 , is a gammaherpesvirus and the proposed etiologic agent of Kaposi's sarcoma, primary effusion lymphoma, and a subset of multicentric Castleman's diseases [19]. KSHV infects a variety of human cell types, including B, T, endothelial, epithelial, fibroblast, and keratinocyte cells, as well as nonhuman cell types, including owl monkey kidney and baby hamster kidney fibroblast cells, in some cases resulting in the establishment of long-term cultures [20-22]. As Kaposi's sarcoma is mainly a vascular tumor and the spindle-shaped cells composing the vasculature in Kaposi's sarcoma express certain endothelial cell markers, it is believed that this type of tumor originates from
KSHV-infected endothelial cells. The present study assessed the kinetics of DcR 3 mRNA and soluble DcR3 protein expression in KSHV-infected HUVECs. In KSHVinfected HUVECs, DcR3 mRNA expression showed a biphasic expression pattern; the first peak was observed around $10 \mathrm{~h}$ postinfection and then decreased around $16 \mathrm{~h}$ postinfection. After the viral gene was actively expressed, the expression of DcR3 increased again. The two peaks of expression may be caused by different underlying mechanisms. According to a previous study, little KSHV mRNA is expressed until about $10 \mathrm{~h}$ postinfection, after which expression gradually increases. The second increase in DcR3 expression in KSHV-infected HUVECs could be a response to viral replication or to the expression of viral proteins, whereas the first peak occurred before viral genes were actively expressed. Although the exact mechanism of upregulation of DcR3 expression prior to active viral gene expression was not investigated in detail in this study, the first phase of induction could be due to a receptor-mediated event during virus entry. The cellular response to virus entry or the presence of proteins or microRNAs derived from the viral capsid would have an effect on the expression of DcR3.

The results of the present study show that expression of DcR3 has a protective effect against KSHV infection in HUVECs. Exposure of KSHV-infected cells to antagonistic anti-DcR3 antibodies resulted in a cytotoxic effect in over $50 \%$ of cells, which TUNEL and DNA fragmentation assays showed was mediated by apoptosis. DcR3 is known to inhibit apoptosis by blocking FasL, LIGHT and TL1A. Although FasL is known to be involved in apoptosis, endothelial cells are resistant to Fas-mediated cell death after exposure to soluble FasL or agonist anti-Fas antibody [23]. Preliminary experiments in the present study did not show an effect of soluble FasL on HUVECs (data not shown). When the expression of TL1A and LIGHT were investigated in KSHV or mock-infected HUVECs, only TL1A mRNA was detected and there was no amplification of LIGHT at postinfection time $24 \mathrm{~h}$ (fig. 6a). Therefore, LIGHT would not act on HUVECs in an autocrine manner. As DcR3 was reported to induce cell proliferation, migration, MMP-2 expression, and angiogenesis by neutralizing TL1A autocrine in HUVECs, the increase in the expression of soluble DcR3 during KSHV infection would inhibit apoptosis of HUVECs in a similar way. Although there was no significant difference of TL1A mRNA expression between KSHV- and mock-infected cells (fig. 6b), a recent study reported that most of the TL1A expressed in HUVECs was in an uncleaved membrane-bound form and that a very small amount of TL1A 
Fig. 5. Anti-DcR3 antibody-induced apoptosis in KSHV-infected HUVECs. KSHVinfected cells treated with anti-DcR3 antibody or control IgG were subjected to TUNEL and DNA fragmentation assays to evaluate apoptosis. A TUNEL staining of control IgG treated KSHV-infected HUVECs (a) and anti-DcR3 antibody treated cells (b). B Cleavage of cellular DNA in KSHV-infected HUVECs. Cellular DNA was extracted from anti-DcR3 antibody treated $(+)$ and control IgG-treated cells $(-)$. DNA fragmentation was analyzed by agarose gel electrophoresis. Molecular weight markers are indicated in the leftmost lanes.

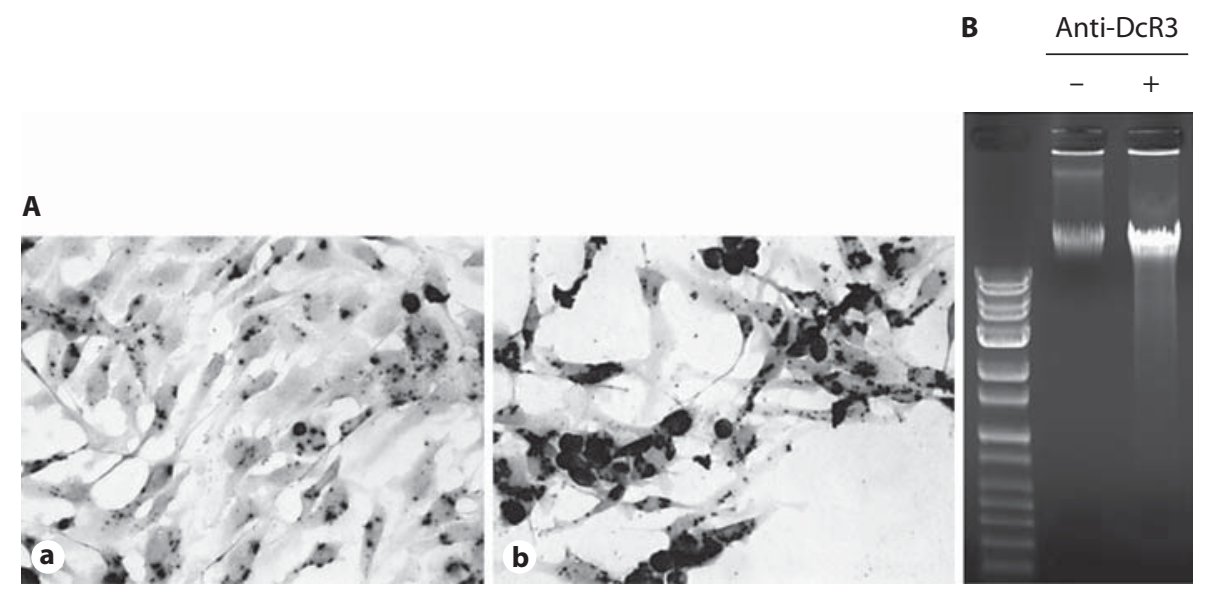

was expressed as two functionally distinct isoforms of the soluble fragment [15]. Hence, mRNA expression analysis was not helpful for the analysis of the expression of soluble TL1A. As one of the soluble fragments of TL1A has been reported to be an inducer of apoptosis in HUVECs, it is possible that DcR3 blocks this soluble TL1A on HUVECs during the early phase of KSHV infection. Further studies will be required to elucidate the exact mechanism of DcR3-mediated apoptosis inhibition.

Induction of pro-angiogenic and pro-inflammatory cytokines, such as IL-6, Ang-2, MMP-1, MMP-2 and MMP-9, has been described in KSHV-infected HUVECs. These proteins are thought to have roles in the pathogenesis of KSHV-related lesions, such as Kaposi's sarcoma. Some of these proteins induce angiogenesis by increasing cell invasiveness $[24,25]$. DcR3 could protect KSHV-infected endothelial cells by inhibiting apoptosis at the early phase of KSHV infection. One outcome of protecting cell death is to allow more time for the virus to replicate, and make more virons to spread to other cells. Additionally, other paracrine-dependent suppressive effects for apoptosis might also be relevant under in vivo conditions. As one ligand of DcR3 is TL1A, it could affect the angiogenesis of KSHV-infected endothelial cells. DcR3 might have similar effects on the pathogenesis of Kaposi's sarcoma in the presence of other induced cytokines.

In summary, the present results show that DcR3 was expressed in HUVECs during the early phases of KSHV infection. DcR3 showed a protective effect against virusmediated cytotoxicity in KSHV-infected HUVECs. These results suggest that DcR3 could play a role in the establishment and maintenance of KSHV infection in HUVECs.

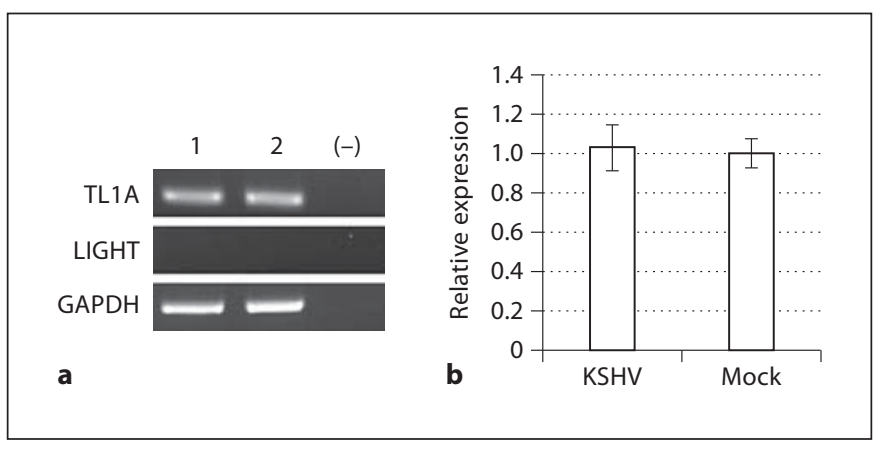

Fig. 6. mRNA expression of TL1A and LIGHT in KSHV- or mockinfected HUVECs. At $24 \mathrm{~h}$ postinfection, total RNA was collected from KSHV- or mock-infected HUVECs, and each mRNA was measured by RT-PCR. a RT-PCR of TL1A and LIGHT from KSHV- or mock-infected HUVECs. Lane 1 and 2 show the amplified RT-PCR products from KSHV-infected HUVECs and mockinfected HUVECs, respectively. Lane (-) was a template-free negative control. b Quantitative, real-time RT-PCR for TL1A. Quantification of TL1A mRNA levels from KSHV- or mock-infected HUVECs was normalized to GAPDH expression. These results represent the means of triplicate experiments. Standard deviations are indicated with error bars.

\section{Acknowledgement}

This work was supported by the Korea Research Foundation (KRF) grant of the Korea government (MEST) (No. 20090069011). 


\section{References}

$>1$ Roth WK, Brandstetter H, Stürzl M: Cellular and molecular features of HIV-associated Kaposi's sarcoma. AIDS 1992;6:895-913.

$\checkmark 2$ Chang Y, Cesarman E, Pessin MS, Lee F, Culpepper J, Knowles DM, Moore PS: Identification of herpesvirus-like DNA sequences in AIDS-associated Kaposi's sarcoma. Science 1994;266:1865-1869.

3 Boshoff C, Weiss RA: Epidemiology and pathogenesis of kaposi's sarcoma-associated herpesvirus. Philos Trans R Soc Lond B Biol Sci 2001;356:517-534.

4 Yang C-R, Hsieh S-L, Teng C-M, Ho F-M, Su W-L, Lin W-W: Soluble decoy receptor 3 induces angiogenesis by neutralization of TLla, a cytokine belonging to tumor necrosis factor superfamily and exhibiting angiostatic action. Cancer Res 2004;64:1122-1129.

$\checkmark 5$ Yu KY, Kwon B, Ni J, Zhai Y, Ebner R, Kwon BS: A newly identified member of tumor necrosis factor receptor superfamily (TR6) suppresses light-mediated apoptosis. J Biol Chem 1999;274:13733-13736.

6 Pitti RM, Marsters SA, Lawrence DA, et al: Genomic amplification of a decoy receptor for fas ligand in lung and colon cancer. Nature 1998;396:699-703.

7 Takahama Y, Yamada Y, Emoto K, Fujimoto H, Takayama T, Ueno M, Uchida H, Hirao S, Mizuno T, Nakajima Y: The prognostic significance of overexpression of the decoy receptor for Fas ligand (DcR3) in patients with gastric carcinomas. Gastric Cancer 2002;5: 61-68.

$>8$ Ibrahim SM, Ringel J, Schmidt C, Ringel B, Muller P, Koczan D, Thiesen HJ, Lohr M: Pancreatic adenocarcinoma cell lines show variable susceptibility to trail-mediated cell death. Pancreas 2001;23:72-79.

-9 Arakawa Y, Tachibana O, Hasegawa M, Miyamori T, Yamashita J, Hayashi Y: Frequent gene amplification and overexpression of decoy receptor 3 in glioblastoma. Acta Neuropathol 2005;109:294-298.
10 Wu Y, Han B, Sheng H, Lin M, Moore PA, Zhang J, Wu J: Clinical significance of detecting elevated serum DcR3/Tr6/M68 in malignant tumor patients. Int J Cancer 2003; 105:724-732.

11 Roth W, Isenmann S, Nakamura M, Platten M, Wick W, Kleihues P, Bahr M, Ohgaki H, Ashkenazi A, Weller M: Soluble decoy receptor 3 is expressed by malignant gliomas and suppresses cd95 ligand-induced apoptosis and chemotaxis. Cancer Res 2001;61:27592765.

12 Ho CH, Hsu CF, Fong PF, Tai SK, Hsieh SL, Chen CJ: Epstein-barr virus transcription activator RTA upregulates decoy receptor 3 expression by binding to its promoter. J Virol 2007;81:4837-4847.

13 Ohshima K, Haraoka S, Sugihara M, Suzumiya J, Kawasaki C, Kanda M, Kikuchi M: Amplification and expression of a decoy receptor for fas ligand (DcR3) in virus (EBV or HTLV-I) associated lymphomas. Cancer Lett 2000;160:89-97.

14 Muck C, Herndler-Brandstetter D, Micutkova L, Grubeck-Loebenstein B, Jansen-Durr P: Two functionally distinct isoforms of TL1a (TNFSF15) generated by differential ectodomain shedding. J Gerontol A Biol Sci Med Sci 2010;65:1165-1180.

15 Yoo SM, Zhou FC, Ye FC, Pan HY, Gao SJ: Early and sustained expression of latent and host modulating genes in coordinated transcriptional program of $\mathrm{KSHV}$ productive primary infection of human primary endothelial cells. Virology 2005;343:47-64.

16 Gao SJ, Deng JH, Zhou FC: Productive lytic replication of a recombinant Kaposi's sarcoma-associated herpesvirus in efficient primary infection of primary human endothe lial cells. J Virol 2003;77:9738-9749.

17 Zhou FC, Zhang YJ, Deng JH, Wang XP, Pan HY, Hettler E, Gao SJ: Efficient infection by a recombinant Kaposi's sarcoma-associated herpesvirus cloned in a bacterial artificial chromosome: application for genetic analysis. J Virol 2002;76:6185-6196.
18 Yoo SM, Ahn AK, Seo T, Hong HB, Chung MA, Jung SD, Cho H, Lee MS: Centrifugal enhancement of Kaposi's sarcoma-associated virus infection of human endothelial cells in vitro. J Virol Methods 2008;154:160-166.

19 Moore PS, Chang Y: Molecular virology of Kaposi's sarcoma-associated herpesvirus. Philos Trans R Soc Lond B Biol Sci 2001;356: 499-516.

20 Cerimele F, Curreli F, Ely S, Friedman-Kien AE, Cesarman E, Flore O: Kaposi's sarcomaassociated herpesvirus can productively infect primary human keratinocytes and alter their growth properties. J Virol 2001;75: 2435-2443.

21 Ciufo DM, Cannon JS, Poole LJ, Wu FY, Murray P, Ambinder RF, Hayward GS: Spindle cell conversion by Kaposi's sarcoma-associated herpesvirus: formation of colonies and plaques with mixed lytic and latent gene expression in infected primary dermal microvascular endothelial cell cultures. J Virol 2001;75:5614-5626.

22 Flore O, Rafii S, Ely S, O’Leary JJ, Hyjek EM, Cesarman E: Transformation of primary human endothelial cells by Kaposi's sarcomaassociated herpesvirus. Nature 1998;394: 588-592.

23 Muck C, Herndler-Brandstetter D, Micutkova $L$, Grubeck-Loebenstein B, Jansen-Durr P: Two functionally distinct isoforms of TL1A (TNFSF15) generated by differential ectodomain shedding. J Gerontol A Biol Sci Med Sci;65:1165-1180.

-24 Kang T, Ye FC, Gao SJ, Wang LD: Angiogenesis, Kaposi's sarcoma and Kaposi's sarcoma-associated herpesvirus. Virol Sin 2008; 23:449-458.

25 Qian LW, Xie J, Ye F, Gao SJ: Kaposi’s sarcoma-associated herpesvirus infection promotes invasion of primary human umbilical vein endothelial cells by inducing matrix metalloproteinases. J Virol 2007;81:70017010 . 